\title{
From Mobile to Wearable System: A Wearable RFID System to Enhance Teaching and Learning Conditions
}

\author{
Souad Larabi Marie-Sainte, ${ }^{1}$ Muna S. Alrazgan, ${ }^{1}$ Fatiha Bousbahi, \\ Sanaa Ghouzali, ${ }^{1}$ and Wadood Abdul ${ }^{2}$ \\ ${ }^{1}$ Information Technology Department, College of Computer and Information Sciences, King Saud University, \\ Riyadh 11543, Saudi Arabia \\ ${ }^{2}$ Department of Computer Engineering, College of Computer and Information Sciences, King Saud University, \\ Riyadh 11543, Saudi Arabia \\ Correspondence should be addressed to Sanaa Ghouzali; sghouzali@ksu.edu.sa
}

Received 26 February 2016; Accepted 7 April 2016

Academic Editor: Miltiadis D. Lytras

Copyright (c) 2016 Souad Larabi Marie-Sainte et al. This is an open access article distributed under the Creative Commons Attribution License, which permits unrestricted use, distribution, and reproduction in any medium, provided the original work is properly cited.

\begin{abstract}
Over the last decade, wearable technology has seen significant developments, making it possible to enhance our lives. One of the areas in which wearable technology can cause large changes is education, where it can be used to make educational experiences intrinsically motivating and more relevant to youth culture. In this paper, we focus on the use of wearable technology to improve the educational environment. The quantity of electronic assets used in the learning environment is rising, which presents a managerial problem when these devices are nonfunctioning. Therefore, we present a mobile application to solve this problem. The suggested approach consists of creating a mobile application named classroom clinic (CRC) to help faculty members and students locate the closest maintenance technician via wearable radio frequency identification (RFID) technology and to provide fast responses to the problems alerted to in the classroom, thereby avoiding any disturbances or delays during the lecture. Moreover, this application allows the maintenance service to efficiently manage any malfunctions of classroom electronic devices. To evaluate the CRC application, a pilot study was conducted at the College of Computer and Information Sciences, female campus of King Saud University, with 15 faculty members and students and 5 clinic members. The results showed high usability rates and generally positive attitudes towards using the app.
\end{abstract}

\section{Introduction}

The world is becoming more educated and technology is driving the change to make our lives easier, safer, healthier, and more convenient. One of the areas in which technology is having an incredible impact is wearable technology. Wearable technology refers to mobile and lightweight advanced electronic devices that are incorporated into clothing and accessories. These wearable devices are designed to provide means for gathering quantitative information regarding the wearer's everyday activities and surroundings. Over the last decade, technologies such as augmented reality and ubiquitous computing have enabled large advances in the field of wearable technology.
A growing collection of wearable devices is appearing in the marketplace. For example, the Sony SmartWatch has become commonplace, allowing users to organize notifications, check their email, and perform other productive tasks using a basic interface. Jawbone UP is a wristband device that allows users to monitor their physical activity, including the number of steps taken and the number of calories burned. Google Glass is one of the latest technologies; it enables users to see information regarding their surroundings. An example of successful testing of wearable technology in the GCC is the use of Google Glass to track traffic violations in Dubai [1]. Moreover, the Dubai Police Department is planning to use Google Glass to detect criminals using facial recognition software installed on Google Glass [2]. However wearable 
technology is still in its infancy and several challenges, such as battery life, display size, cost, design, and lack of privacy, are reducing widespread adoption and availability of wearable devices [3]. Seeing the endless exciting uses we can make of these wearable devices, we believe technology will improve and the constraints that developers need to address and overcome now may not be a problem in the near future.

The existing wearable devices can be deployed to develop a wide variety of applications and systems. In the healthcare sector, wearable devices are particularly useful to monitor the wellbeing and fitness levels of patients and, thereby, lower overall healthcare costs $[4,5]$. In education, wearable technology can help students and faculty to more efficiently interact with each other and with their surroundings; however, currently, there are few concrete educational applications [6]. For example, using mobile devices, students can visit and explore any part of the world using a virtual reality application named Expedition, which was developed by Google [7]. Google Glass can be used as a tool for enhancing student studying and providing access to pertinent information. In the field of medical education, wearable technology has been successfully applied [3]; specifically, Google Glass has been tested in medical and surgical education, where it showed great value for improving students' practice and skills [8]. Other wearable devices can also be used in the educational environment; for example [9], smart jewelry or other accessories could alert students working in chemical laboratories to hazardous conditions. Wearable cameras can allow the learner to engage simultaneously as observer and reporter while instantly capturing hundreds of photographs or data regarding an offsite trip, which can be later accessed via email or other online applications. Smart watches enable users to view and organize notifications.

In modern educational institutions, the use of electronic devices is becoming a necessity. However, these devices can become defective and require prompt maintenance to avoid disturbances during the lecture. The principle explored in this study is enhancing learning by supporting and maintaining the learning environment. Our aim is to develop a mobile application to manage the malfunctioning of classroom electronic materials via radio frequency identification (RFID) tag technology. The application allows the support service to automatically determine which classroom contains the defective electronic materials and to immediately receive and manage all calls for maintenance. The system combines RFID tag technology with mobile computing to track the support service members and the relevant classroom (the classroom in which a malfunction occurred).

The remainder of this paper is organized as follows. Section 2 describes studies related to the application of RFID tags to education. The proposed case study is presented in Section 3. The system design and evaluation are reported and discussed in Sections 4 and 5, respectively. Section 6 concludes the paper and presents a number of directions for future work.

\section{Related Works}

2.1. RFID Technology: An Overview. Most wearable technology applications rely on some form of wireless communication to send sensor data to an information gateway such as a mobile phone or personal computer, which then transmits the data to a remote site over the Internet. Wireless communication may be roughly divided into three categories based on range and data volume. Wi-Fi represents the longest range and the highest data rates. Bluetooth is a mid-range and medium-data-rate system. Short-range and low-data-rate solutions are represented by radio frequency identification (RFID) and near field communications (NFC). In this study, we focus on the use of RFID in an educational environment.

The term RFID includes all of the technologies that use radio waves to automatically identify and track objects, animals, or people [10]. RFID technology operates using a reader and a transmitter, that is, the RFID reader (also called transceiver) and the RFID tag. The reader and tag communicate with each other using an antenna integrated with each of the two components. The reader transmits energy to the tag via radio waves; then, it sends a request for information to the tags located within the range of its magnetic field. Once the data is transmitted to the RFID reader, it converts the data into radio waves and forwards them to the appropriate RFID software. The tags are composed of a chip (which may contain memory) and a radio frequency antenna. There exist two main types of tags: active (which require a power source) and passive (batteryless) tags. Passive tags have a long lifetime and are quite small (varying in size from a grain of rice to a blue card badge format). The RFID chip can be read at a distance that can vary depending on the reader and the tag used, typically from $1 \mathrm{~cm}$ to $200 \mathrm{~m}$. The tags can be embedded within several items: packaging, clothing labels, plastic cards, and books [11].

RFID systems are becoming increasingly prominent in our daily life. The worldwide turnover from the sale of components and the provision of services related to RFID technology has increased by $40 \%$ per year since 2005 [12]. In fact, there are few economic sectors that remain untouched by the RFID revolution. Consequently, many sectors envisage combining RFID with barcodes; in the near future, barcodes will likely be replaced by RFID.

RFID systems have many advantages over barcode systems. First, the stored information can be updated at any time using a single RFID reader-encoder. Second, these systems can identify many items (up to several thousand) simultaneously, thereby saving time. Third, by employing radio frequencies to communicate, RFID systems are able to identify objects without requiring line of sight. Moreover, novel RFID technologies have brought costs down and several standards are being developed.

2.2. RFID Applications in Education. In the education sector, RFID technology can enhance the teaching and learning conditions, simplify processes and procedures, and save time. However, despite a large body of existing RFID based systems in different areas such as identification, transportation, healthcare, and welfare [13], there is far less literature 
addressing RFID application in education. Thus even after many years in the market, the use of RFID in education is still in the experimental stage. This is due, on one hand, to a lack of trust in this technology in terms of privacy and security [14] and, on the other hand, to the high cost of tag production. Nevertheless, in using new RFID technology, we are witnessing the emergence of mature RFID applications that can reduce costs, increase revenue, and drive competitive advantages [15]. Therefore, recently, research into and development of educational-platformbased RFID technology have increased; the potential applications for RFID systems will result in large-scale deployment (indeed, the investigation of RFID technology in educational environments is needed). We can classify the applications based on RFID technology into two categories: applications that facilitate the learning environment and applications to teaching and learning platforms. We will present some of these applications below.

Musex [16] is a system for supporting children's collaborative learning in a museum. Using handheld devices (PDAs), children explore the museum and answer questions related to exhibitions. Using RFID technology, the system locates a child's position in the museum. An RFID tag reader/writer and a wireless LAN card are installed in each device. An RFID tag is also installed at each exhibition. In an experiment in a museum, thirty-five children (aged five to twelve) participated and 13 questions were provided to encourage exploration of the exhibitions. The system allows users to test their knowledge of the exhibitions. The system was used by a pair of pupils: each child was given a PDA and a transceiver, which allowed the pupils to communicate with each other and collaboratively solve questions. In their paper, the authors reported several positive interactions of pupils using the system: they found that the users paid more attention to less attractive and less interactive exhibitions and that the children were more engaged with the learning activities.

In [17], the authors present a wireless pedestrian navigation system that includes a wearable computing solution. The system assists blind students to make their way around a campus. The system uses an RFID grid based on passive, low-cost, high-frequency RFID tags. The grid is installed under the flooring and used to convey the precise location and detailed attributes regarding the surrounding areas on the campus. On the campus, RFID tags are placed along outdoor pathways, in building halls, and in rooms; they store information about their surrounding space. The RFID reader is integrated into the user's shoe and walking cane, with a Bluetooth connection to the student's cell phone. The system was tested for visually impaired or blind users. The RFID system caused no fatal errors in navigation and good results were obtained. The authors will provide a new version in the future.

In [18], the authors developed an outdoor education support system using two technologies: RFID and QR (quick response), a two-dimensional kind of barcode to provide location awareness. In their paper, the authors found that traditional computing has low levels of mobility and embeddedness. They proposed a system that combines mobile computing (high mobility but low embeddedness) and pervasive computing (low mobility but high embeddedness). An experiment has been performed involving 21 students divided into four groups during a university course in the field of natural vegetation. Each group had a PC with an RFID reader and a mobile phone with a QR code reader. A total of 42 tag pairs were placed at various locations across a horticultural farm and forest of a nonflat area of roughly 5 ha. After this investigation, the students were asked to fill in a questionnaire to rate the features provided by the system using a fivepoint Likert scale from 1 (lowest) to 5 (highest). The results reported by the authors showed that the students were not very comfortable with the mobile screen's display of images; however, overall, the students enhanced their understanding and motivation to learn.

TangiSense $[19,20]$ is a table that provides tangible interactions with objects using RFID technology to capture the context (user and object, etc.). The objects can be virtual or physical and are placed on the surface of the table. The table is fitted with RFID sensors to detect tangible objects (equipped with RFID tags). The virtual objects can be manipulated by the user via a glove with RFID tags, allowing objects to follow the user's movements. In this project, the researchers applied the context awareness principle to an interactive table with RFID technology. An experiment involving 24 subjects was conducted and studies compared information captured using RFID technology and that without it. The findings showed that RFID can be used as an input to applications. The prototype allows exploring new areas of research and providing a platform for educational games.

In [21], RFID technology is used to solve exam timetabling problem in universities. The authors developed a web based application to schedule different exams in the same halls simultaneously. Student's identity card is embedded with RFID tag reader connected to computers located in common areas of the university. By doing so, the system allows the student to get information about exam details (exam day, hour, and hall). The system was evaluated using 10 students and 8 exams, showing good results.

RFID panic buttons system is a real time location system [22] designed to support school-safety and emergencyresponse procedures and practices within several schools in the USA. It is a Wi-Fi based RFID tag. A teacher wears a badge with the RFID tag embedded in it and, in the situation of any type of emergency, draws down on the badge's switch in order to alert colleagues. When an alert is issued, the software captures and interprets the emergency's location. The software diffuses a message directly to the appropriate badges inside the district, thereby informing other personnel where the emergency is occurring.

Many middle and high schools in the United States and UK are adopting RFID systems to track their students. Some of them provided their students with RFID tag tied to a lanyard, while others are requiring them to wear a sweatshirt with an RFID embedded tag [23, 24].

Moreover, to curb gun violence in schools, authorities in the USA required an RFID chip to be implemented in all public schools by 2015 using the student's smartphone devices [25]. The program was approved by educators after 
the successive rash of shootings that left several people dead and many others injured each time.

Additionally, in [26], the authors proposed an RFID based system to supervise access to different campus areas (library, laundry, restaurant, laboratory, etc.). The system uses two types of reader: fixed reader and mobile reader and both of them are connected to a PC server. The fixed readers are present in each point of access and communicate with the server and with every other terminal in the network. The mobile reader consists of a PDA in which a reader has been embedded. The authors specified several capabilities of this system, especially its speed in processing data coming from thousands of transponders at thousands of access points.

\section{Case Study}

Most educational institutions and universities use high technology to accomplish different tasks, especially in learning contexts. Learning has always been of primary importance. Educational institutions and universities are integrating technology in classrooms via mobile computing, projectors, interactive whiteboards, television broadcasts, and so forth. These resources require maintenance as soon as a problem occurs, because delays in the maintenance process may disrupt both the students and the faculty members, interrupt their work, and waste their time.

Usually, institutions and universities provide maintenance services. However, most faculty members face challenges when a problem occurs, because they do not know (or have forgotten) which maintenance service is responsible for addressing a given malfunction. In addition, faculty members and/or students might not know the location of maintenance services, particularly if there is more than one maintenance service office. Moreover, it is difficult for the maintenance service members to recall all classrooms problems and their locations, especially when they receive several calls at the same time. Consequently, many teachers experience wasted time when a tool in their classroom is nonfunctional. Calling and waiting for technician intervention can take more time than expected. Knowing that, for a lecture of only fifty minutes, any wasted time can have significant negative effects on student learning achievement.

The goal of our classroom clinic (CRC) mobile application is to provide an efficient solution that can serve faculty members, students, and maintenance service staff members. This solution combines RFID tag technology with mobile computing; it consists of creating a mobile application that provides faculty members or students with a fast maintenance response to the problem alerted to. This application allows the maintenance service member (called the classroom clinic member) to accurately manage all malfunctions of classroom electronic materials. There is a great deal of evidence that using wearable technology, such as our RFID system, can solve this problem. As we will see in the testing phase of our system, faculties reported positive effects more frequently. The system helped to reduce stress and disruption to the class.

The CRC application is an interactive map implemented on the Android platform; it uses the Internet or Wi-Fi in addition to RFID tag technology. Figure 1 displays the main components of an RFID system. This system consists of RFID tags, locaters, readers, a switch, a server, and mobile phones to display the result of the detected tags. The tags contain electronically stored information. In this study, active RFID tags were used. Each user (faculty or student), classroom, classroom clinic, and classroom clinic member should have one tag. As shown, the mobile application communicates with the server to request information regarding the RFID tags. The server sends requests to the switch, which communicates with the RFID reader via the antenna. The RFID reader provides the connections between the tag data and the enterprise system software (in this case, the mobile app) that needs the information. As a result, the RFID reader collects data requested from tags. The RFID reader can detect the RFID tag at a given distance (approximately 100 meters). Then, the locator specifies the detected tag from among the (multiple) tags that one reader can access. The mobile application CRC is illustrated in detail below.

Figure 2 shows the main interface of the CRC mobile app. When the user starts the application she can change the language (Arabic/English) and select the page that is related to her status (user or member). The main features of this application derive from RFID tag technology. This technology enables the achievement of several users and system requirements. For instance, it allows users to track classroom clinic members, find the shortest path to the classroom clinic and/or to the concerned classroom (where the malfunction has occurred) from the current position, and indicate all of the concerned classrooms on the map with red dots.

Because time is very important for fixing problems in the classroom, the CRC system obtains the shortest path on the map. We used the Dijkstra algorithm [27] to determine the shortest path on the map. This algorithm is known to solve the single-source shortest path problem. For a given source node in the graph, the algorithm determines the optimal route. It finds the path with the lowest cost between that node and every other node in the graph. The algorithm can also find the shortest path from a given node to a given destination node. The Dijkstra algorithm that we implemented in our system is illustrated as follows.

\section{Dijkstra Algorithm Used in the CRC APP}

$\operatorname{Dist}[s] \leftarrow 0$ (distance to source node is zero)

Do Dist $[n] \leftarrow \infty$ (set all other nodes to infinity)

$S \leftarrow\{\}$ (S, the set of visited nodes is initially empty)

$Q \leftarrow N$ (Q, the queue initially contains all nodes)

While $(Q \neq\{\})$ (while the queue is not empty)

Do $u \leftarrow \operatorname{Min} \operatorname{dis}(Q$, dist) (select the element of $Q$ with the min distance)

$S \leftarrow S \cup\{u\}$ (add $u$ to list of visited nodes)

For all $n=$ neighbors $[u]$

Do if $\operatorname{dist}[n]>\operatorname{dist}[u]+w(u, n)$ (if new shortest path found, $w$ is the weight of the edge $(u, n))$

Then $d[n] \leftarrow d[u]+w(u, n)$ (set new value of the shortest path)

Return dist. 


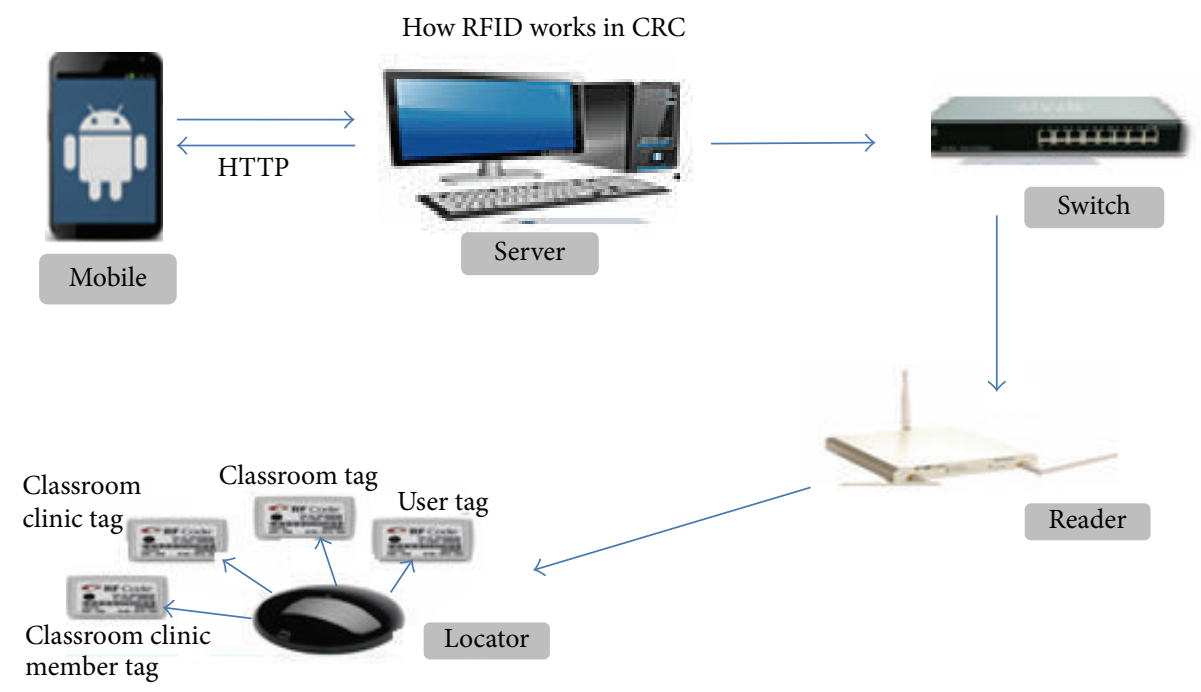

FIGURE 1: Combination of the RFID tag technology with CRC application.

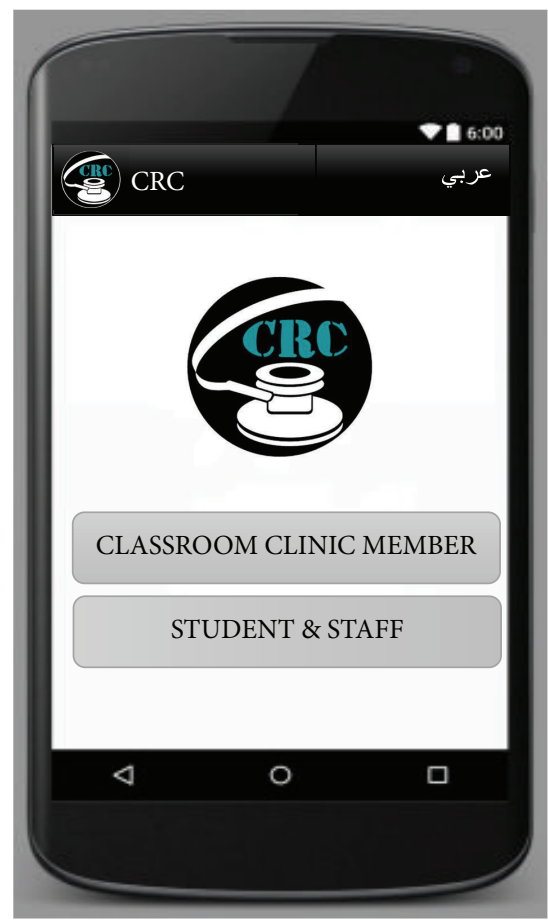

FIgURE 2: Main interface of the CRC mobile app.

The most important part is finding the shortest path in the map between the requested node and the CRC clinic node so as to improve the response time of our CRC system.

\section{System Design}

To implement our system, we evaluated the requirements mentioned in the previous section and designed the system prototype. Next, the system was implemented as a mobile web application using RFID technology. Figure 3 presents the $\mathrm{CRC}$ conceptual diagram. This figure presents the important classes of CRC systems and their relationships. The CRC app consists of the following classes: CRC, CRC map, CRC member, user, classroom, location, repertory problem, classroom clinic, and RFID class. For example, the CRC class communicates with the following classes: the CRC map class of the given building, the members (clinic personnel), and classroom clinic information (such as name, office number, services, and office phone number) that is used when the user requests service. The RFID class detects the location of the classrooms, classroom clinic, classroom clinic members, and users using their ID tags for easy tracking and indicating 


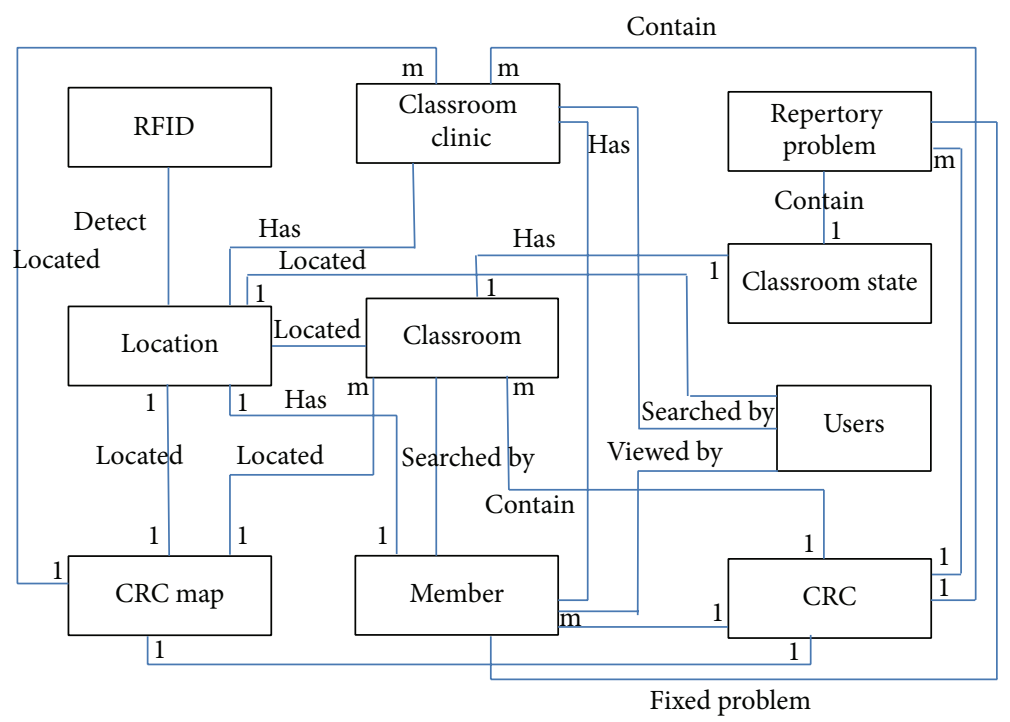

FIgURE 3: Conceptual diagram.

the technician location to the faculty member via the interactive map. Additionally, the classroom clinic members can easily discover the concerned classroom in which the problem has been reported.

The CRC system stores all of the information related to classroom clinic and classroom clinic members in a centralized database. In addition, it stores the identifiers of the RFID tags related to users, classroom clinics, classroom clinic members, and classrooms so that they can easily be determined. We adopted the client-server [28] system architecture, which corresponds to a straightforward solution to the problem considered. The solution minimizes the cost of modifications and minimizes redundancy of both code and data.

Figure 4 shows the main pages of the CRC app for the admin homepage, the classroom clinic homepage, and the user homepage and their important functions. The design of the interfaces is simple and follows W3C guidelines. The user (a student, a faculty member, or any staff member) can navigate using the CRC to determine her current position and determine the shortest path to a specific classroom clinic. Moreover, she can detect the location of the classroom clinic members and search for classroom clinics and/or classroom clinic members. The main feature is to indicate classrooms on the map (using red dots) in which a problem has occurred, by just reporting this problem to the classroom clinic member. The administrator (admin) user (after logon) has the ability to manage the CRC system for any updates related to the map, manage the CRC clinic members by updating their contact information and their ID tag, and provide any updates related to the CRC app. The classroom clinic member (after logon) can update personal information on her account. The classroom clinic member can find the shortest path on the map to the classroom in which a problem has occurred after receiving the alert (problem report) from the CRC user.

Once the problem is fixed, the classroom clinic member can update the classroom status by changing the red dot to a green dot as shown in Figure 5. Note that the green dot will be removed after 30 minutes.

\section{System Evaluation}

In this section, details regarding the testing process are presented. Our objective is to ensure that the application meets all of the system and user requirements. To do this, we tested CRC at the CCIS (College of Computer and Information Sciences) at the female campus of King Saud University, Riyadh, Saudi Arabia. The new female campus of KSU contains a huge CCIS building that consists of three floors, containing more than 150 classrooms/offices. All of the classrooms, offices, and meeting rooms are furnished with different electronic devices: computers, printers, projectors, smart boards, and televisions. The college has two classroom clinics, e-learning and distance learning, and a computer unit.

E-learning and distance learning are under the control of three classroom clinic members, all of whom are responsible for responding to calls of failures of televisions, smart boards, terraces, and meeting rooms. They repair all of the faults of electronic devices. The computer unit is under the control of eight members who are responsible for responding to calls of failures in labs. Their tasks are cleaning computers of old files, repairing the printer, checking the Internet connectivity, providing computers for employees, and downloading required programs.

To perform testing of the CRC app, we applied performance testing and user acceptance testing.

5.1. User Acceptance Testing. The purpose of user acceptance testing is to evaluate the CRC system in terms of provided functionality and design convenience. This test also obtains more accurate prediction of the usability and effectiveness of the application. In addition, user acceptance testing ensures that the system satisfies the user and the system requirements. 


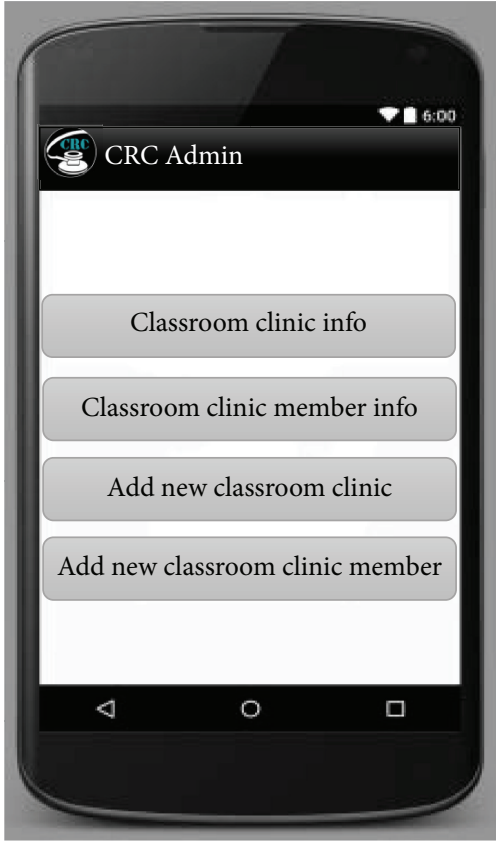

(a)

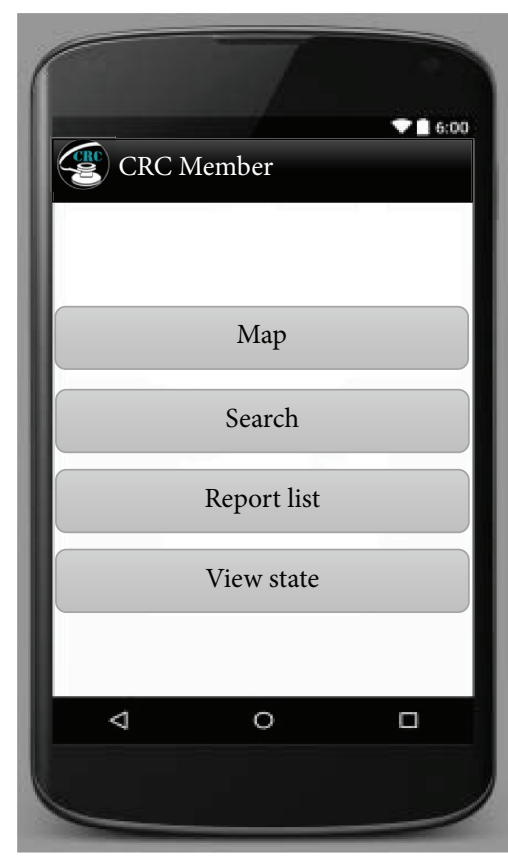

(b)

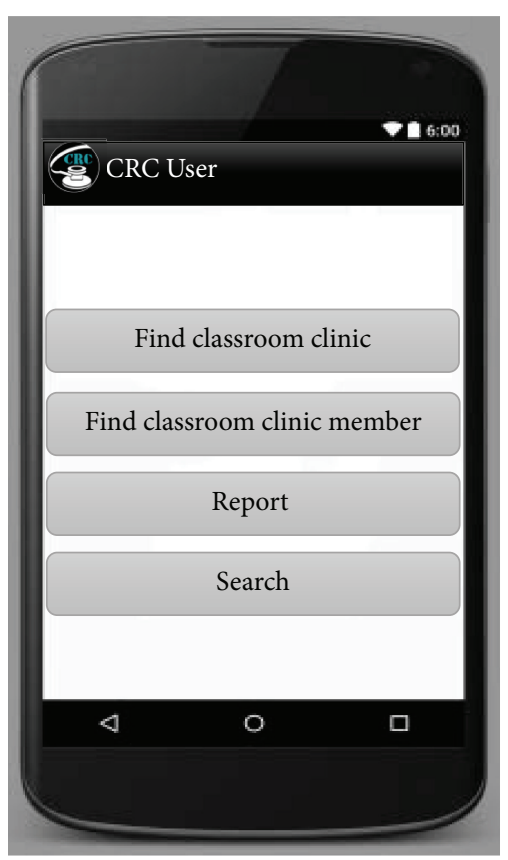

(c)

Figure 4: The admin homepage (a), the classroom clinic member homepage (b), and the user homepage (c).

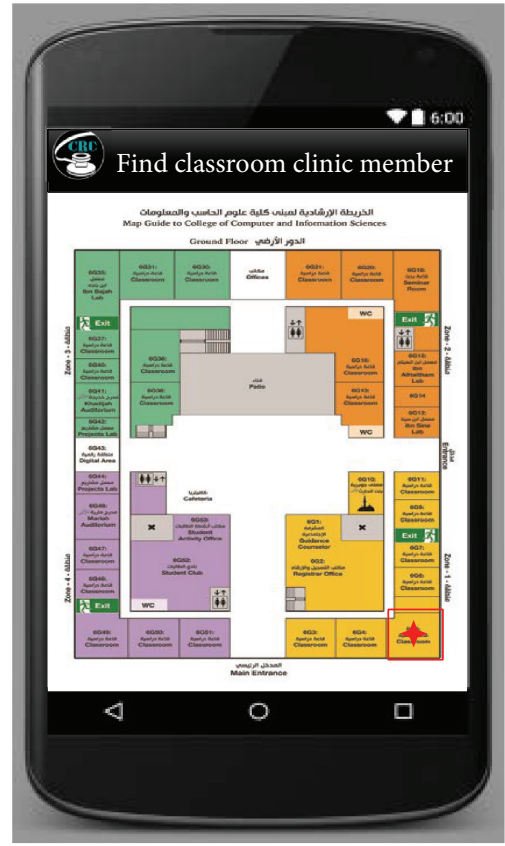

(a)

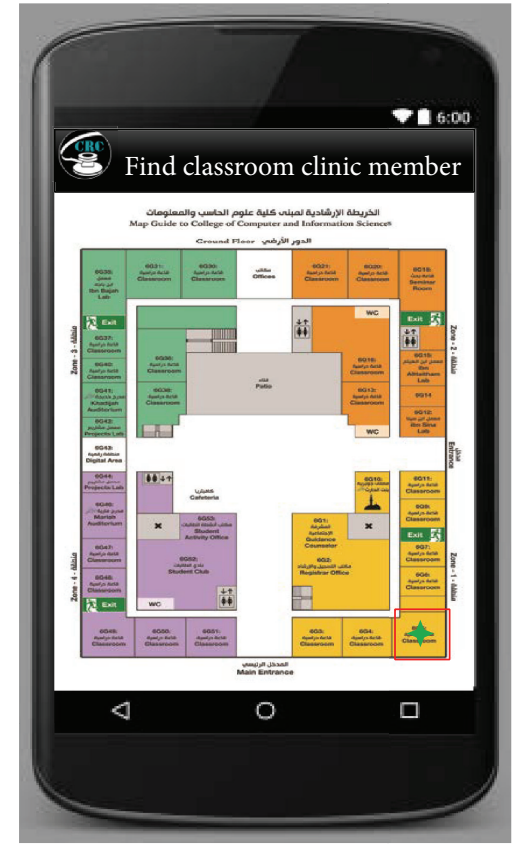

(b)

FIGURE 5: CRC interactive map, indicating the classroom with a red dot when a problem occurs (a) and green dot when the problem is solved (b).

We have developed a holistic test case scenario to evaluate the functionalities available to all categories of CRC users. Table 1 illustrates the classroom clinic member, faculty member, and administrator test case functions. Note that all the associated functions have passed successfully (indicated by tick). The scenario is described as follows: the faculty member is in a classroom, opens the CRC app, and displays the map. The map indicates the location of the classroom clinic, as well as the classroom clinic members who are connected to the CRC app. When the faculty member reports 
TABLE 1: Acceptance test.

\begin{tabular}{|c|c|c|c|}
\hline Task & User & CRC member & Admin \\
\hline (1) Login & & $\checkmark$ & $\checkmark$ \\
\hline (2) Logout & & $\checkmark$ & $\checkmark$ \\
\hline (3) Find classroom & $\checkmark$ & $\checkmark$ & $\checkmark$ \\
\hline (4) Find classroom clinic or classroom clinic member & $\checkmark$ & $\checkmark$ & $\checkmark$ \\
\hline (5) Display shortest path & $\checkmark$ & $\checkmark$ & $\checkmark$ \\
\hline (6) Detect the current location & $\checkmark$ & $\checkmark$ & $\checkmark$ \\
\hline (7) Send report & $\checkmark$ & $\checkmark$ & \\
\hline (8) Search on map & $\checkmark$ & $\checkmark$ & $\checkmark$ \\
\hline (9) Report list & & $\checkmark$ & \\
\hline (10) Delete report & & $\checkmark$ & \\
\hline (11) View state & $\checkmark$ & $\checkmark$ & $\checkmark$ \\
\hline (12) Download reports & & $\checkmark$ & \\
\hline (13) Switch language & $\checkmark$ & $\checkmark$ & \\
\hline (14) Forgot password & & $\checkmark$ & $\checkmark$ \\
\hline (15) Ask for help & & $\checkmark$ & \\
\hline (16) Add classroom clinic or classroom clinic member & & & $\checkmark$ \\
\hline (17) Edit classroom clinic or edit classroom clinic member & & & $\checkmark$ \\
\hline (18) Delete classroom clinic or delete classroom clinic member & & & $\checkmark$ \\
\hline
\end{tabular}

a device failure, the indicator of the classroom on the map turns to a red dot. At the same time, one of the classroom clinic members intercepts the alert and finds the concerned classroom (indicated in red) on the map.

After selecting the shortest path option, the path from the classroom clinic member's current location to the concerned classroom appears on the map. Once the problem is fixed, the classroom clinic member updates the classroom state and the indicator color resets to green. Another scenario concerns the search function: the faculty member is in her office, and she faces a problem with her computer or printer. She can search for either a specific classroom clinic member name or the classroom clinic office that can fix the problem. When the search is performed the related information about the classroom clinic and/or the classroom clinic member is displayed.

We also evaluated the functionality of the CRC system for the administrator. As shown in the third column in Table 1, the admin logs into the system, views the report on the system, and then manages the database by adding/deleting/updating a new classroom clinic and/or a new classroom clinic member. The admin also can perform any function available to a CRC user.

To assess the performance of our proposed system, we evaluated the system for 10 faculty members and students, 5 classroom clinic members, and 2 admins at CCIS. We focused on calculating the average time (AVT) the users took to perform a task and the average number of errors (AVE) the users made when achieving a task. In this test, we measured the usability of our system in terms of effectiveness, efficiency, and satisfaction. The effectiveness is measured based on the number of errors that the user made when performing the different tasks. The results from the classroom clinic member and the faculty member/students indicated high usability rates (AVE does not exceed 2 for each task). Furthermore, we measured the efficiency in terms of the time the user took when performing a specified task. Most of the tasks were done in insignificant time, except searching using the maps, which took approximately $30 \mathrm{~s}$. Therefore, the CRC system is efficient. Satisfaction is measured in terms of user and system requirement satisfaction. According to CCIS users, CRC meets all of their needs; they expressed a desire to use it in the future.

5.2. Performance Testing. Performance testing concerns performing a series of tests until the application performance reaches an unacceptable value. We used an Android Assistant tool [29] to determine the CRC's performance. Figure 6 shows the CPU and the RAM use before and during running the CRC system. The CRC requires only $20 \%$ of the CPU and 37 $\mathrm{MB}$ of RAM, which is acceptable.

\section{Conclusion}

RFID tag technology is a well-known wearable technology that can be efficiently involved in education, where its functioning can be simplified and changed. RFID is mainly used in enhancing learning by helping to improve learning methods and/or cultivating competency. In this paper, we used RFID to enhance learning by supporting and maintaining the student's environment. This environment is assumed to be all the electronic technology devices that the students use for learning. We have presented the design and development of classroom clinic (CRC), a mobile application to track support service staff members and defective classrooms using the RFID tag technology. To the best of our knowledge, the proposed application is the first wearable RFID system to enhance teaching and learning conditions. The use of 


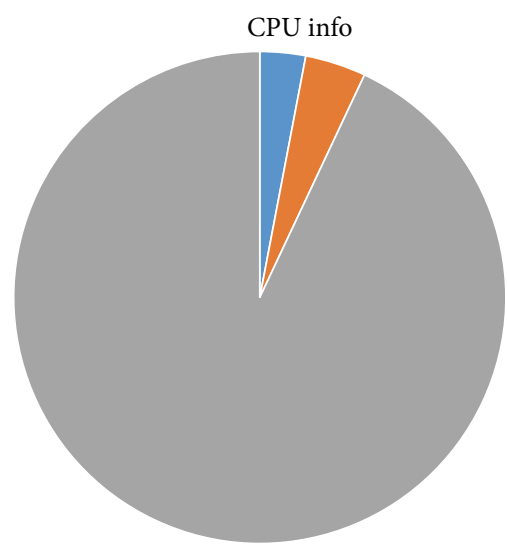

System 3\%

User $4 \%$

Idle $93 \%$

(a)

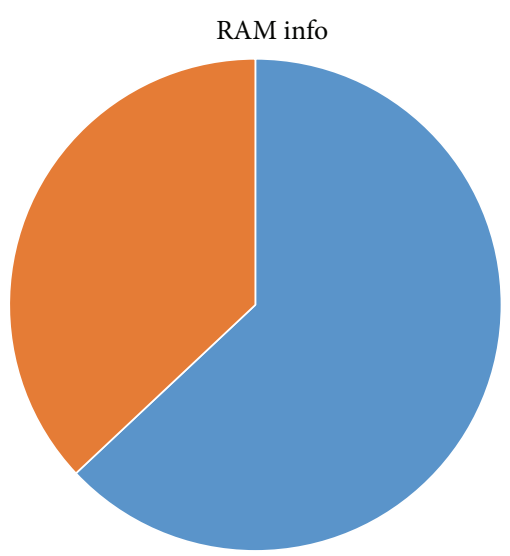

Used: $1.1 \mathrm{~GB}$

- Free: $662.92 \mathrm{MB}$

Total: $1.74 \mathrm{~GB}$

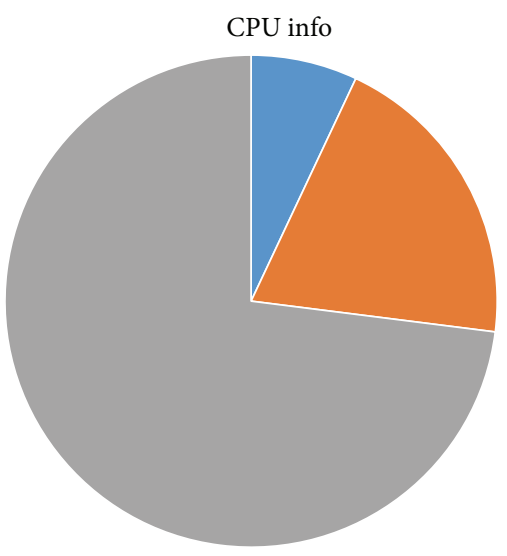

System: $7 \%$

User: $20 \%$

Idle: $73 \%$

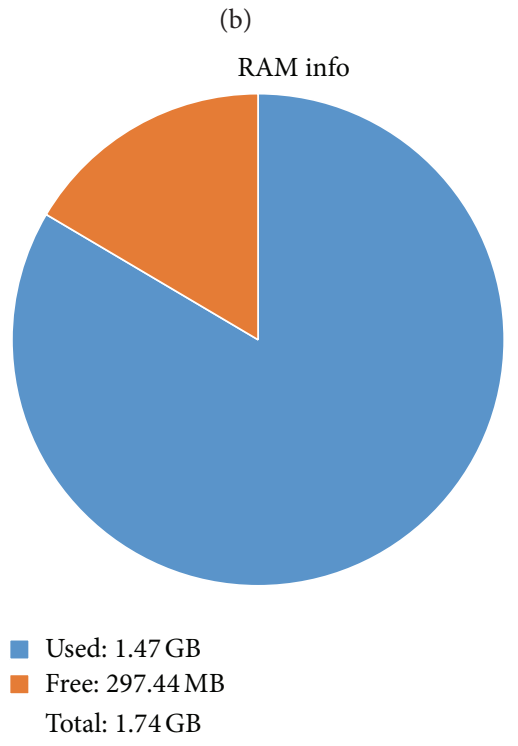

(d)

FIGURE 6: Before and during running CRC system.

RFID in our application highly improves the maintenance services. In fact, all the information about classrooms and offices is stored in the RFID which mainly facilitates the access to the classrooms where the problem has occurred and/or classroom clinic members. In addition, using RFID for communication is greatly recommended as it can easily identify objects and thus save time. This proposed application was successfully implemented and provided satisfactory results. In fact, faculty reported several positive effects of the system, namely, reducing stress and disruption to the classroom. The application has a straightforward and userfriendly interface that does not require high computing skill. The application fulfills the general requirements of various educational institutions, especially those of CCIS. Different tests have proven that CRC is an efficient system that promptly responds to maintenance requests, meets faculty's needs, and achieves its intended purpose. This system can be used by different educational institutions.

One object of future work is to implement this system in different OS environments. Moreover, the application can be improved by implementing it for all of the floors of a building at the same time, rather than viewing only one selected floor.

\section{Competing Interests}

The authors declare that there are no competing interests regarding the publication of this paper.

\section{Acknowledgments}

This research project was supported by a grant from the "Research Center of the Female Scientific and Medical 
Colleges," Deanship of Scientific Research, King Saud University. The first author thanks M. Albogami, A. Alkhuraijy, A. Alshnefi, A. Alotaibi, A. Alshehri, and A. Almansur for their effort in designing and implementing the CRC app.

\section{References}

[1] L. Eadicicco, "Cops In Dubai Are Using Google Glass To Catch Speeding Drivers," Business Insider, May 2014, http://www.businessinsider.com/police-using-google-glass-2014-5.

[2] "Dubai police plan to deploy Google Glass facial recognition," Biometric Technology Today, vol. 2014, no. 11, p. 12, 2014.

[3] N. Sultan, "Reflective thoughts on the potential and challenges of wearable technology for healthcare provision and medical education," International Journal of Information Management, vol. 35, no. 5, pp. 521-526, 2015.

[4] S. Patel, H. Park, P. Bonato, L. Chan, and M. Rodgers, "A review of wearable sensors and systems with application in rehabilitation," Journal of NeuroEngineering and Rehabilitation, vol. 9, article 21, 2012.

[5] R. K. Kumar, "Technology and healthcare costs," Annals of Pediatric Cardiology, vol. 4, no. 1, pp. 84-86, 2011.

[6] B. Petrovic, "Wearable computing in education," ICT Management, vol. 849, pp. 934-941, 2014.

[7] J. Holland, "Announcing expeditions: taking students places a school bus can't go," Official Google for Work Blog, May 2015, http://googleforwork.blogspot.com/2015/05/announcing-Expeditions-taking-students-places-a-school-bus-cant-go.html.

[8] O. Moshtaghi, K. S. Kelley, W. B. Armstrong, Y. Ghavami, J. Gu, and H. R. Djalilian, "Using Google Glass to solve communication and surgical education challenges in the operating room," Laryngoscope, vol. 125, no. 10, pp. 2295-2297, 2015.

[9] L. Johnson, S. Adams Becker, M. Cummins, V. Estrada, A. Freeman, and H. Ludgate, NMC Horizon Report: 2013 Higher Education Edition, The New Media Consortium, Austin, Tex, USA, 2013.

[10] K. Finkenzeller, RFID Handbook: Fundamentals and Applications in Contactless Smart Cards and Identification, John Wiley \& Sons, New York, NY, USA, 2003.

[11] M. Ward, R. Van Kranenburg, and G. Backhouse, "RFID: frequency, standards, adoption and innovation," Tech. Rep., JISC Technology and Standards Watch, 2006.

[12] "Applications et perspectives de la rfid," August 2012, http:// www.culturemobile.net/quotidien-intelligent/applications-etperspectives-rfid.

[13] K. Jung and S. Lee, "A systematic review of RFID applications and diffusion: key areas and public policy issues," Journal of Open Innovation: Technology, Market, and Complexity, vol. 1, pp. $1-9,2015$.

[14] A. Juels, "RFID security and privacy: a research survey," IEEE Journal on Selected Areas in Communications, vol. 24, no. 2, pp. 381-394, 2006.

[15] M. Bhuptani and S. Moradpour, RFID Field Guide: Deploying Radio Frequency Identification Systems, Prentice Hall PTR, Upper Saddle River, NJ, USA, 2005.

[16] K. Yatani, M. Sugimoto, and F. Kusunoki, "Musex: a system for supporting children's collaborative learning in a museum with PDAs," in Proceedings of the 2nd IEEE International Workshop on Wireless and Mobile Technologies in Education, pp. 109-113, IEEE, March 2004.
[17] S. Willis and A. Helal, "RFID information grid and wearable computing solution to the problem of wayfinding for the blind user in a campus environment," in Proceedings of the 9th Annual IEEE International Symposium on Wearable Computers, Osaka, Japan, October 2005.

[18] N. Osawa, K. Noda, S. Tsukagoshi et al., "Outdoor education support system with location awareness using RFID and symbology tags," Journal of Educational Multimedia and Hypermedia, vol. 16, no. 4, pp. 411-428, 2007.

[19] S. Kubicki, S. Lepreux, C. Kolski, C. Perrot, and J. Caelen, "TangiSense: présentation d'une table interactive avec technologie RFID permettant la manipulation dobjets Tangibles et Traçables," in Proceedings of the 21ème Conférence de l'Association Francophone sur l'Interaction Homme-Machine, pp. 351354, Grenoble, France, October 2009.

[20] S. Kubicki, S. Lepreux, and C. Kolski, "Evaluation of an interactive table with tangible objects: application with children in a classroom," in Proceeding of the 2nd Workshop on Child Computer Interaction "UI Technologies and Educational Pedagogy", in Conjunction with CHI 2011 Conference, Vancouver, Canada, May 2011.

[21] A. Akbulut and G. Yılmaz, "University exam scheduling system using graph coloring algorithm and RFID technology," International Journal of Innovation, Management and Technology, vol. 4, no. 1, pp. 66-72, 2013.

[22] B. Bachelor, "New Indiana school district implements Ekahau RFID panic buttons," RFID Journal, February 2014, http://www .rfidjournal.com/articles/view?11431.

[23] K. W. Leisner, Student safety through the utilization of radio frequency identification (RFID) within the education system [M.S. of Science Cybersecurity Intelligence], ProQuest LLC, Ann Arbor, Mich, USA, 2014.

[24] T. Omarzu, "RFID Chips to be Used on Chattooga County Buses to Track Students," July 2014, http://www.timesfreepress.com/ news/local/story/2014/jul/14/tracking-students-rfid-chips-tobe-used-on-buses/252137.

[25] "RFID Chip Implemented in All Public Schools by 2015-Effort to Curb Gun Violence," June 2014, http://nationalreport.net/rfid-chip-implemented-public-schools-2015-effort-curb-gun-violence/\#sthash.xHzgCi3p.dpuf.

[26] C. E. Turcu, C. Turcu, V. Popa, and V. Gaitan, "ICT and RFID in education: some practical aspects in campus life," http://arxiv .org/abs/1503.04286.

[27] T. Bhatt, "Application of graph theory to find optical paths between two nodes," International Journal of Research in Humanities \& Social Sciences, vol. 2, no. 8, pp. 10-15, 2014.

[28] J. Jing, A. S. Helal, and A. Elmagarmid, "Client-server computing in mobile environments," ACM Computing Surveys, vol. 31, no. 2, pp. 117-157, 1999.

[29] W. Mfg, "Assistant for Android," https://play.google.com/store/ apps/details?id=com.advancedprocessmanager. 

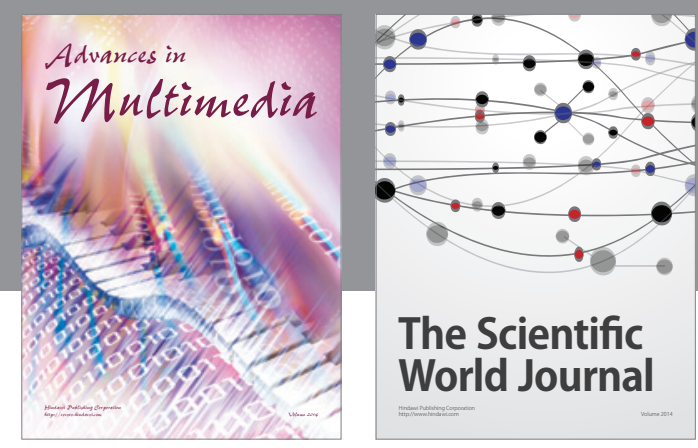

The Scientific World Journal
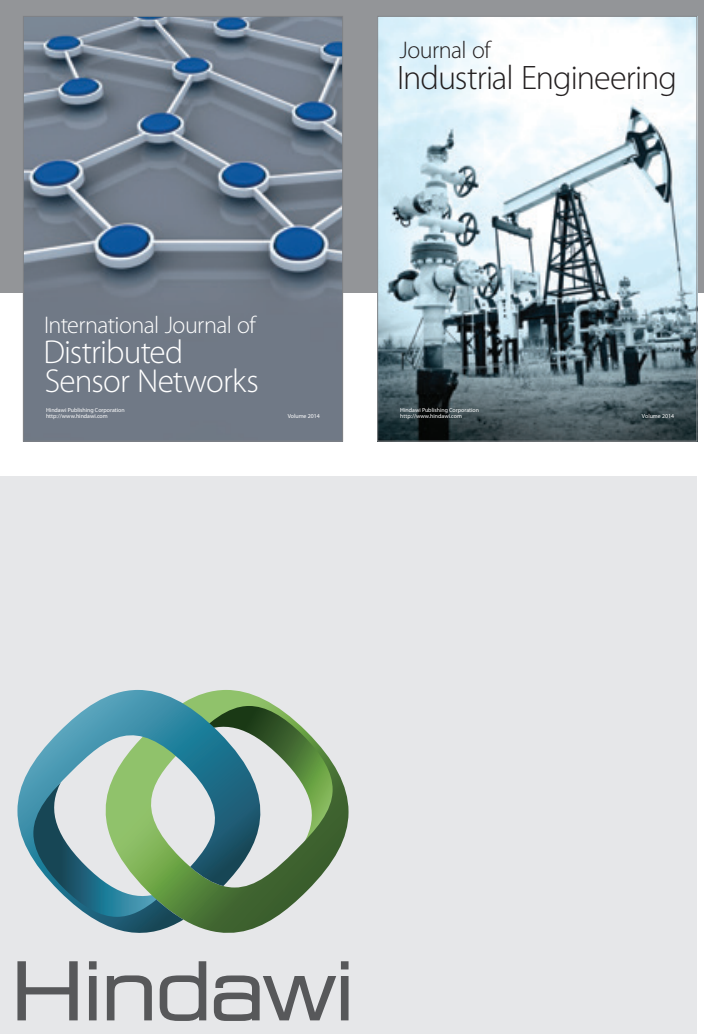

Submit your manuscripts at

http://www.hindawi.com

\section{Computer Networks} and Communications
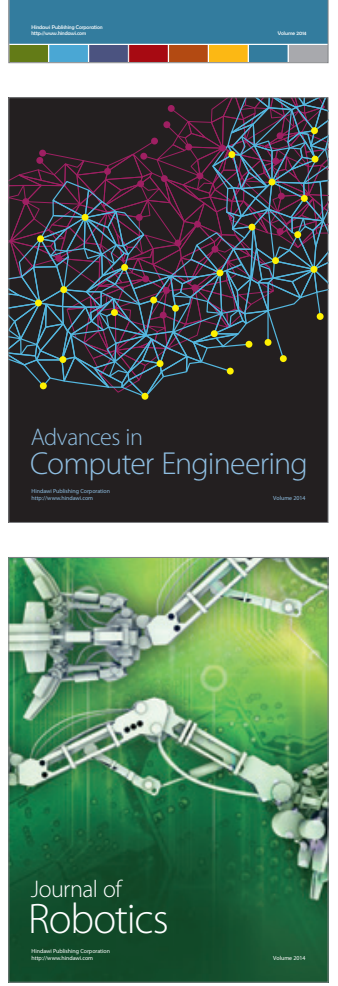
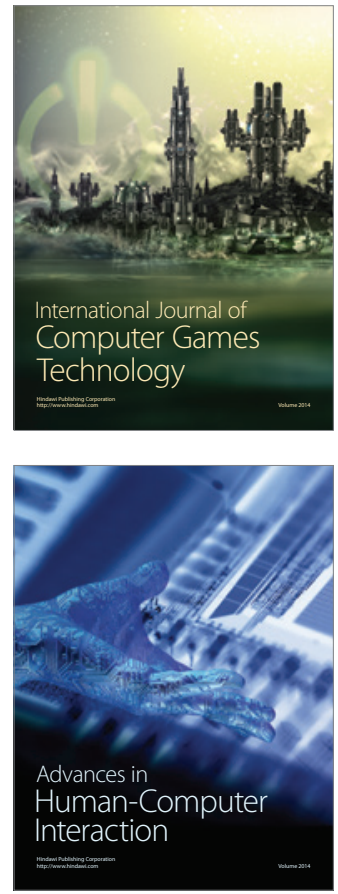
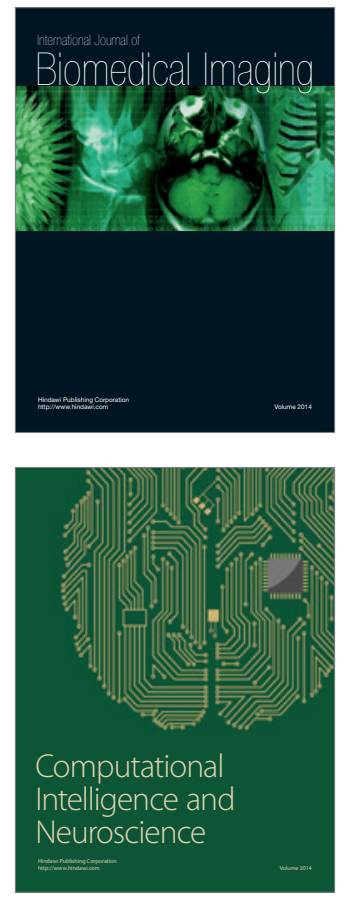
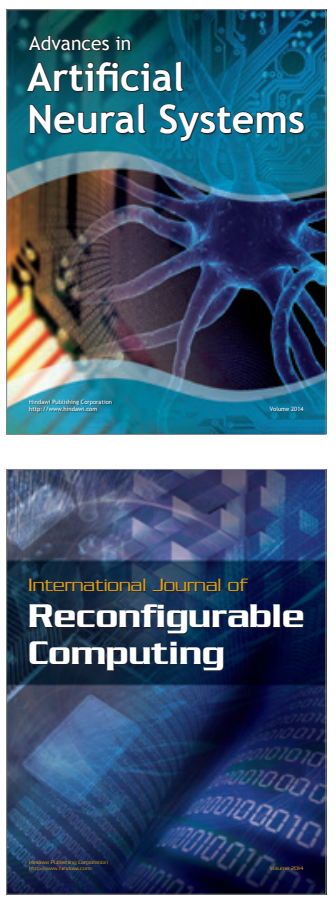
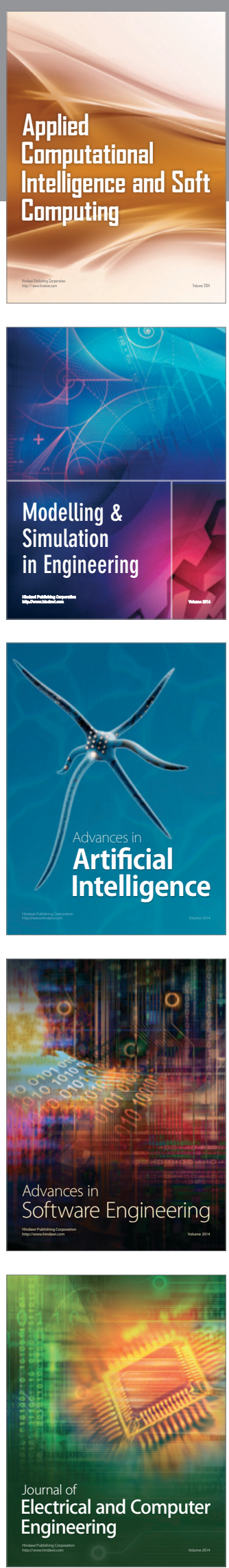